\title{
МЕТОД АДАПТИВНОГО КОНТРОЛЯ НЕУСТОЙЧИВОЙ ДИНАМИКИ В ДВУХЭЛЕМЕНТНЫХ СИСТЕМАХ С КОНКУРЕНЦИЕЙ НА ПРИМЕРЕ ДУОПОЛИИ
}

Одной из актуальных проблем при моделировании систем и процессов является исследование динамики функционирования систем с конкуренцией, в частности, то, что при определенных условиях в них появляется нежелательная неустойчивая динамика, которая в течение длительного времени может привести к непредсказуемому поведению элементов системы. Математические модели типа Курно-Пу дают возможность проанализировать такие процессы, поскольку учитывают реальные условия функционирования упомянутых выше систем с конкуренцией.

При применении DFC-метода, с целью стабилизации неустойчивого поведения систем с конкуренцией, целесообразно его сравнение с аналогичными методами, в частности, с методом адаптивного контроля - АС-методом, принцип действия которого заключается в предположении, что ожидания элементов системы изменяются от “наивных" к “адаптивным". Оптимальное значение исходной характеристики элемента системы в последующий период зависит от значения этой характеристики в текущий период, а также от средневзвешенного ожидаемого значения этой же исходной характеристики конкурирующего элемента, тоже в текущий период.

Проведенные исследования позволяют сделать вывод, что в двухэлементных системах с конкуренцией для стабилизации хаотической динамики в системе целесообразно применять только одну управляющую функцию. Такое управление быстрее приводит систему в состояние равновесия, по сравнению с применением двух управляющих функций, т.е. попытки двух элементов одновременно стабилизировать систему. Именно поэтому применение метода адаптивного контроля, как и применение DFC-метода к структуре системы, также считается методом “индивидуального" контроля в неустойчивой системе.

Исследованные модели управления можно использовать не только для стабилизации неустойчивой динамики на олигополистических рынках, но и во всех системах, элементы которых взаимосвязаны и конкурируют между собой относительно общего ограниченного ресурса.

\footnotetext{
1 Dr hab. inż. Natalia Iwaszczuk, AGH University of Science and Technology, Faculty of Management, Krakow (Д.э.н. Наталия Иващук, Факультет управления, ГорноМеталлургическая Академия, Краков)

2 Д.э.н., профессор Жанна Поплавская, Факультет управления, Жешовская политехника им.И.Лукасевича, Жешов

3 К.т.н. Ирина Кавалец, Институт прикладной математики и фундаментальных наук, Национальный университет “Львовская политехника”, Львов
} 
Ключевые слова: система с конкуренцией, дуополия, модель Курно-Пу, точка равновесия, неустойчивая динамика, метод адаптивного контроля.

\section{1.ВВЕДЕНИЕ}

Одной из актуальных проблем при моделировании систем и процессов является исследование динамики функционирования сложных систем, в частности систем с конкуренцией. В структуре технической, производственной либо информационной системы с конкуренцией часто выступает несколько (от 2 до 10) конкурирующих элементов, при этом лишь незначительная часть из них обеспечивает доминирующую долю результата функционирования всей системы. Появление в этой системе новых конкурирующих элементов утруднено либо невозможно. Наиболее очевидным примером сложной системы с конкуренцией является система олигополии (олигополистического рынка), которая состоит из фирм, производящих аналогичные продукты и конкурирующих между собой.

Как было проанализировано и описано в статье Иващук и Кавалец ${ }^{4}$, а также в монографии Костробия и др. ${ }^{5}$, наиболее часто к системам с конкуренцией применяются модели типа Курно-Пу, с изоэластичной (нелинейной) функцией ожидаемых значений исходных характеристик элементов системы и различными постоянными значениями параметров их предельного поведения ${ }^{6}$. Исходными характеристиками для олигополии могут быть как объемы выпуска продукции, так и цена изделий. Модель Курно-Пу рассматривает первый случай, а потому именуется моделью количественной олигополии. Основными параметрами предельного поведения элементов для олигополистического рынка являются предельные издержки фирм.

Одной из важных проблем функционирования таких систем является то, что при определенных условиях в них появляется нежелательная неустойчивая динамика, которая в течение длительного времени может привести к непредсказуемому поведению элементов системы, и поэтому требует применения соответствующих методов управления, с целью ее стабилизации ${ }^{7}$. Математические модели типа Курно-Пу дают возможность проанализировать такие процессы, поскольку учитывают реальные условия функционирования упомянутых выше систем с конкуренцией. Построению обобщенной модели систем с конкуренцией, в

\footnotetext{
${ }^{4}$ N. Iwaszczuk, I. Kavalets, Application of mathematical models in the study of oligopolistic markets, [w:] Zastosowania modeli matematycznych w ekonomii, finansach i bankowości, red. P. Pusz. Wydawnictwo Uniwersytetu Rzeszowskiego, Rzeszów 2012, s. 27-47.

5 П.П. Костробій, І.В. Алексєєв, І.Б. Хома, Б.В. Гнатів, І.І. Кавалець, В.І. Алексєєв, Математичні моделі регулювання фінансових потоків, ред. П.П. Костробій, Растр-7, Львів 2012.

${ }^{6} \mathrm{Cm}$. A. Cournot, Recherches sur les principes mathématiques de la théorie des richesses, Paris: Hachette 1838

${ }^{7}$ T. Puu, Chaos in duopoly pricing, "Chaos, Solitons \& Fractals" 1 (1991), s. 573-581.

A. Matsumoto, Controlling the Cournot-Nash Chaos, "Journal of Optimization Theory and Applications" 128/2 (2006), s. 379-392.

L. Chen, G. Chen, Controlling chaos in an economic model, "Physica A" 374 (2007), s. 349-358.

A. Jakimowicz, Stability of the Cournot-Nash equilibrium in standard oligopoly, "Acta Physica Polonica A"121/2-B (2012), s. B-50-B-53.
} 
частности олигополии, а также исследованию условий устойчивости точки ее равновесия были посвящены работы ${ }^{8}$.

Проблемы контроля неустойчивой динамики в рассматриваемых системах были предметом научных исследований ряда публикаций. Так, в работах 9 рассмотривается применение метода управления с обратной связью с задержкой (delayed feedback control method, DFC-метода), с целью управления нестабильным поведением в двухэлементной системе с конкуренцией, с использованием модели Курно-Пу. В результате были установлены значения коэффициентов обратной связи для DFC-метода, при которых система стабилизируется за наименьшее количество временных шагов. На основе проведенных в этих работах исследований было также показано, что применение DFC-метода с двумя управляющими функциями (т.е. с возможностью контроля нестабильных колебаний обоими элементами одновременно) является неэффективным. Обобщение DFC-метода для многоэлементных систем на основе моделей Курно-Пу осуществлено в работах ${ }^{10}$.

Однако при применении DFC-метода с целью стабилизирования неустойчивого поведения систем с конкуренцией важным является также его сравнение с другими методами контроля, которые могут применяться к таким системам. Одним из таких методов является метод адаптивного контроля - АC-метод (англ. adaptive control method), который в работе ${ }^{11}$ был применен к двухэлементной системе с конкуренцией (а именно, к дуополии). Однако не были проведены исследования, которые бы дали ответ на вопрос: какими должны быть значения регулирующих параметров этого метода, которые привели бы систему в равновесное состояние в наиболее короткое время? Сравнение полученных результатов с результатами для DFC-метода может дать ответ на вопрос об эффективности применения этих методов в двухэлементных системах на основе выбранных моделей Курно-Пу, что и сделано в данной работе.

\footnotetext{
${ }^{8}$ N. Iwaszczuk, I. Kavalets, Generalized Cournot-Puu oligopoly model and stability of its equilibrium point, [w:] Materiały z konferencji „Zarządzanie Przedsiębiorstwem - Teoria i Praktyka” (Kraków, 22-23 XI 2012), Wydawnictwo AGH w Krakowie, Kraków 2012, s. 1-16.

П.П. Костробій op.cit.

N. Iwaszczuk, I. Kavalets, Oligopolistic market: stability conditions of the equilibrium point of the generalized Cournot-Puu model, "Econtechmod" 2/1 (2013), s. 15-22.

Н.Л. Іващук, Б.В. Гнатів, І.І. Кавалець, Побудова узагальненої моделі олігополії Курно-Пу та дослідження стійкості ї̈ точки рівноваги, „Журнал обчислювальної та прикладної математики" 2/112 (2013), s. 94-104.

${ }^{9}$ N. Iwaszczuk, I. Kavalets, Some features of application the delayed feedback control method to Cournot-Puu duopoly model, "Econtechmod" 2/4 (2013), s. 29-38.

Н. Иващук, И. Кавалец, Математические методы контроля нестабильных колебаний в конкурирующих системах, „Інформаційна безпека” 1 (2013), s. 12-24.

${ }^{10}$ П.П. Костробій op.cit.

N. Iwaszczuk, I. Kavalets, Delayed feedback control method for generalized Cournot-Puu oligopoly model, [w:] Selected Economic and Technological Aspects of Management, red. N. Iwaszczuk. Wydawnictwo AGH w Krakowie, Kraków 2013, s. 108-123.

${ }^{11}$ L. Chen, op. cit.
} 


\section{2. ОСНОВНЫЕ ПРИНЦИПЫ АДАПТИВНОГО КОНТРОЛЯ}

В двухэлементной системе с конкуренцией функционируют лишь два элемента $F_{1}$ и $F_{2}$, с исходными характеристиками $q_{1}$ и $q_{2}$, соответственно. Конкурирующие элементы системы имеют постоянные, но различные значения параметров, характеризующих их предельное поведение. Обозначим эти параметры $c_{l}$ и $c_{2}$. В случае дуополии исходными характеристиками элементов являются объемы выпуска продукции двух фирм ( $q_{1}$ и $\left.q_{2}\right)$, при соответствующих предельных издержках $\left(c_{1}\right.$ и $\left.c_{2}\right)$.

Модель двухэлементной системы с конкуренцией Курно-Пу имеет вид ${ }^{12}$

$$
\begin{aligned}
& q_{1}(t+1)=\sqrt{\frac{q_{2}(t)}{c_{1}}}-q_{2}(t), \\
& q_{2}(t+1)=\sqrt{\frac{q_{1}(t)}{c_{2}}}-q_{1}(t) .
\end{aligned}
$$

Модель (1) была построена на основе предположений Курно и Пу. Предположение Курно заключается в том, что каждый элемент системы ожидает от своего конкурента подобного поведения (т.е. такого же значения характеристик) в текущий период, как и в предыдущем периоде. Такие ожидания в работе ${ }^{13}$ названы "наивными" (англ. naive expectation).

Нетривиальная точка равновесия системы (1) - равновесие Курно (равновесие Нэша) - принимает значения

$$
\begin{aligned}
& q_{1}^{*}=\frac{c_{2}}{\left(c_{1}+c_{2}\right)^{2}}, \\
& q_{2}^{*}=\frac{c_{1}}{\left(c_{1}+c_{2}\right)^{2}}
\end{aligned}
$$

Устойчивость точки равновесия (2) системы (1) детально исследована в ${ }^{14}$, а также в ${ }^{15}$. Установлено, что точка равновесия (2) является устойчивой, если отношение параметров предельного поведения элементов системы находится внутри интервала $1 \leq c_{r}<3+\sqrt{8}$, и неустойчивой, если $3+\sqrt{8} \leq c_{r} \leq 25 / 4$, где $c_{r}=c_{2} / c_{1}\left(\mathrm{~cm} \cdot{ }^{16}\right)$.

\footnotetext{
${ }^{12}$ Cм. также L. Chen, op. cit.

${ }_{13}$ A. Matsumoto, op. cit.

${ }^{14}$ T. Puu, op. cit.

${ }^{15}$ П.П. Костробій op.cit.

N. Iwaszczuk, I. Kavalets, Oligopolistic...

${ }^{16}$ A. Matsumoto, op. cit.

L. Chen, op. cit.
} 
Принцип действия АС-метода заключается в предположении, что ожидания элементов системы изменяются от “наивных" к “адаптивным” ожиданиям (англ. adaptive expectations) $)^{17}$. Оптимальное значение исходной характеристики элемента системы в последующий период зависит от значения этой характеристики в текущий период, а также от средневзвешенного ожидаемого значения этой же исходной характеристики конкурирующего элемента, тоже в текущий период. Динамический процесс (1) при таких предположениях имеет вид

$$
\begin{aligned}
& q_{1}(t+1)=\left(1-\lambda_{1}\right) q_{1}(t)+\lambda_{1}\left(\sqrt{\frac{q_{2}(t)}{c_{1}}}-q_{2}(t)\right), \\
& q_{2}(t+1)=\lambda_{2}\left(\sqrt{\frac{q_{1}(t)}{c_{2}}}-q_{1}(t)\right)+\left(1-\lambda_{2}\right) q_{2}(t),
\end{aligned}
$$

где $\lambda_{1}, \lambda_{2}-$ регулирующие параметры (англ. adjustment parameters) элементов $F_{1}$ и $F_{2}$. Поскольку модифицированный (адаптивный) процесс (3) возвращается к первоначальному (наивному) процессу (1) при $\lambda_{1}=\lambda_{2}=1$ и динамика отсутствует при $\lambda_{1}=\lambda_{2}=0$, то предполагается, что регулирующие параметры принимают промежуточное значение между нулем и единицей: $0<\lambda_{1}, \lambda_{2}<1$.

\section{3. УСЛОВИЯ УСТОЙЧИВОСТИ ТОЧКИ РАВНОВЕСИЯ СИСТЕМЫ ПРИ ПРИМЕНЕНИИ АДАПТИВНОГО КОНТРОЛЯ}

Целью реализации адаптивного регулирования является стабилизация хаотического динамического процесса непосредственно при помощи регулирующего параметра каждого из элементов. Точка равновесия Курно модели (1) является неустойчивой при определенных значениях параметров системы, о чем уже упоминалось выше.

Адаптивный процесс (3) имеет такую же точку равновесия, как и процесс (1) ${ }^{18}$. Для того, чтобы стабилизировать неустойчивый динамический процесс, линеаризуем систему (3) в окрестности точки равновесия (2).

Матрица Якоби адаптивного процесса (3) в точке равновесия Курно имеет вид

$$
J=\left(\begin{array}{cc}
1-\lambda_{1} & \frac{\lambda_{1}\left(c_{2}-c_{1}\right)}{2 c_{1}} \\
\frac{\lambda_{2}\left(c_{1}-c_{2}\right)}{2 c_{2}} & 1-\lambda_{2}
\end{array}\right),
$$

где след и определитель матрицы равны

П.П. Костробій op.cit.

N. Iwaszczuk, I. Kavalets, Oligopolistic...

${ }^{17}$ A. Matsumoto, op. cit

${ }^{18}$ A. Matsumoto, op. cit. 


$$
\begin{aligned}
& \operatorname{trJ}=2-\left(\lambda_{1}+\lambda_{2}\right), \\
& \operatorname{det} J=\left(1-\lambda_{1}\right)\left(1-\lambda_{2}\right)+\frac{\lambda_{1} \lambda_{2}\left(c_{1}-c_{2}\right)^{2}}{4 c_{1} c_{2}} .
\end{aligned}
$$

В соответствии с критерием Рауса-Гурвица, область устойчивости точки равновесия для двухэлементной системы определяется тремя условиями ${ }^{19}$

$$
\begin{aligned}
& \operatorname{det} J>t r J-1, \\
& \operatorname{det} J<1, \\
& \operatorname{det} J>-t r J-1 .
\end{aligned}
$$

Или

$$
\begin{aligned}
& \frac{\left(1+c_{r}\right)^{2}}{4 c_{r}} \lambda_{1} \lambda_{2}>0, \\
& \lambda_{2}>\frac{4 c_{r} \lambda_{1}}{\left(1+c_{r}\right)^{2} \lambda_{1}-4 c_{r}}, \\
& 2\left(2-\lambda_{1}-\lambda_{2}\right)+\frac{\left(1+c_{r}\right)^{2}}{4 c_{r}} \lambda_{1} \lambda_{2}>0,
\end{aligned}
$$

где $c_{r}$ - отношение параметров предельного поведения. Поскольку эта величина положительная, а регулирующие параметры находятся между 0 и 1, то первая и третья из неравностей (7) всегда выполняются.

Таким образом, область устойчивости, как и для системы (1), определяется флатерной границей, то есть $\operatorname{det} J=1$, и изображается так, как показано на рис. 1. Она разделена на три части: светло-серая и две темно-серые области, образованные линиями $\lambda_{2}=c_{r} \lambda_{1}$ и $\lambda_{2}=\left(1 / c_{r}\right) \lambda_{1}$, на которых дискриминант характеристического уравнения равен нулю. Собственные значения матрицы Якоби (4) являются действительными для значений параметров в темно-серой части и комплексными для светло-серой области.

Траектория монотонно (осциляционно) следует к равновесию Курно, когда регулирующие коэффициенты находятся в темно либо светло-серой области.

\footnotetext{
${ }^{19}$ A. Matsumoto, op. cit.

L. Chen, op. cit.
} 


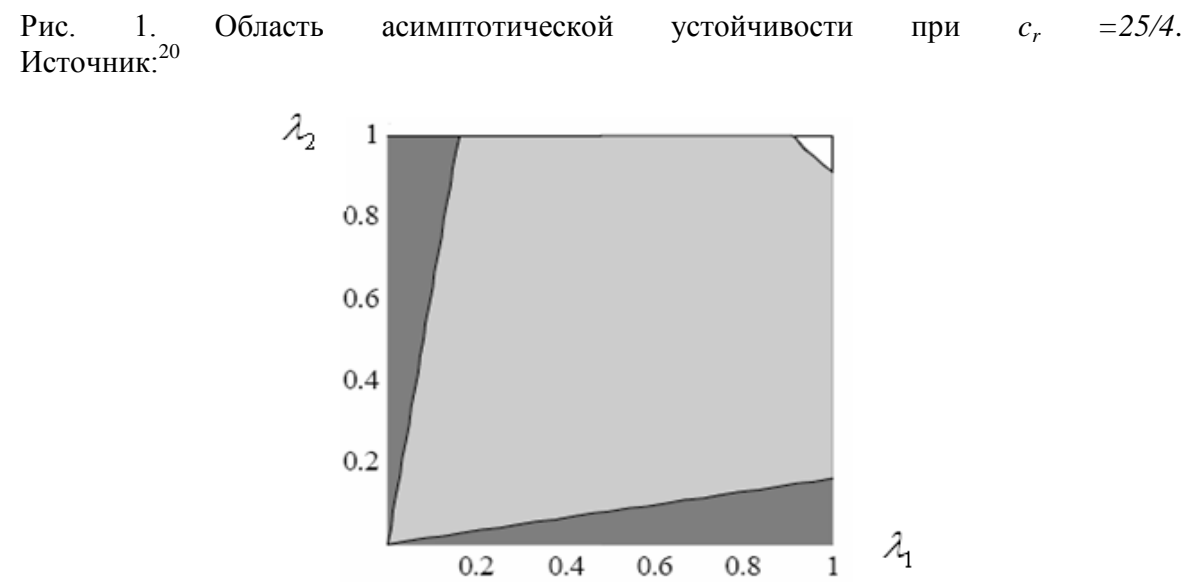

АС-метод не может полностью контролировать хаотическую траекторию, когда адаптивные коэффициенты одновременно близки к 1 (белая область в северовосточной части рис.1).

\section{4. ВЛИЯНИЕ ВЫБОРА РЕГУЛИРУЮЩИХ ПАРАМЕТРОВ НА СКОРОСТЬ УСТАНОВЛЕНИЯ СИСТЕМЫ В ТОЧКЕ РАВНОВЕСИЯ}

В работе ${ }^{21}$ автор провел два численные исследования, со значениями параметров $c_{1}=1, c_{2}=6,25$ и начальными условиями $q_{1}(0)=q_{2}(0)=0,01$. В первом случае, когда были взяты регулирующие параметры со значениями $\lambda_{1}=\lambda_{2}=0,8$, система пришла к точке равновесия за определенное количество временных шагов. Во втором случае, когда регулирующие параметры приняли значения $\lambda_{1}=\lambda_{2}=0,96$, как уже было показано, система продолжала быть неустойчивой (т.е. в ней существовала хаотическая динамика).

В связи с этим возникает следующий вопрос: какие значения регулирующих параметров дает возможность наиболее быстро стабилизировать хаотическую систему при заданном значении ее параметра? Для получения ответа на этот вопрос были проведены численные исследования при таких значениях:

$$
c_{1}=1, c_{2}=6,25, \quad q_{1}(0)=q_{2}(0)=0,01 .
$$

АС-метод начинает действовать с момента времени $t=50$. В первом случае мы выбрали $\lambda_{2}=0,1$, а $0<\lambda_{1}<1$. Во втором - параметры были установлены в значениях: $\lambda_{2}=0,5$ и $0<\lambda_{1}<1$. Графически результат исследования показан на рис. 2 и рис. 3 .

В первом случае были выбраны значения: $\lambda_{2}=0,1$, а $0<\lambda_{1}<1$, а во втором $-\lambda_{2}=0,5$ и $0<\lambda_{1}<1$. Как и в случае применения DFC-метода, было получено необходимое количество временных шагов, которые приводят каждый из элементов системы к равновесному состоянию, т.е. К значению своей исходной характерис-тики

\footnotetext{
${ }^{20}$ A. Matsumoto, op. cit.

${ }^{21}$ A. Matsumoto, op. cit.
} 
$q_{1}{ }^{*}=0,118906$ и $q_{2}{ }^{*}=0,019025$, соответственно. Графически результат исследования показано на рис. 2 и рис. 3.

Было установлено, что в первом случае (при $\left.\lambda_{2}=0,1\right)$ система наиболее быстро приходит в состояние равновесия при $\lambda_{1}=0,6$, что иллюстрирует рис. 2 . Первый элемент достигает равновесного состояния на 81 шаге $\left(t_{l}=81\right.$, т.е. через 31 шаг от начала действия метода), а второй - на 77 шаге $\left(t_{2}=77\right.$, т.е. через 27 шагов от начала действия метода).

Рис. 2. Зависимость количества временных шагов $t$ от выбора регулирующих параметров $\lambda_{1}$ и $\lambda_{2} \quad\left(c_{r}=25 / 4\right): \lambda_{2}=0,1,0<\lambda_{1}<1$. Источник: собственные вычисления

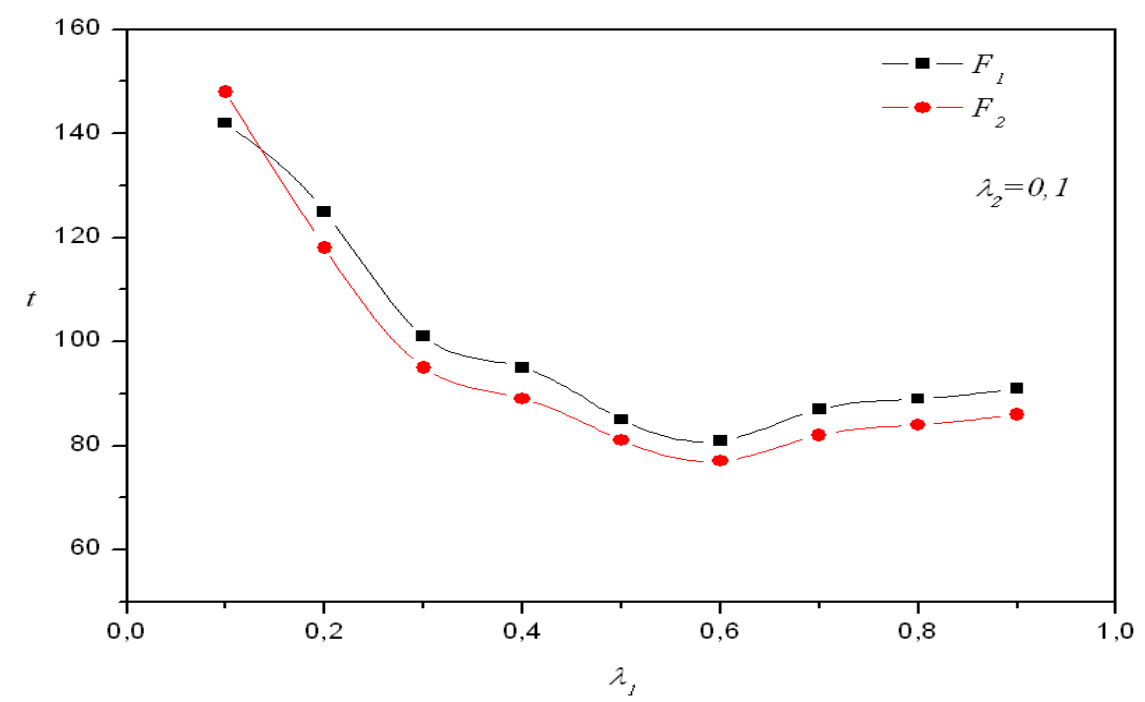

Во втором случае (при $\left.\lambda_{2}=0,5\right)$ система быстро стабилизируется при $\lambda_{l}=0,2$ (см. рис. 3, здесь также $\left.t_{1}=81, t_{2}=77\right)$. Исследуя более детально рис. 3, можно заметить, что при значении $\lambda_{2}=0,5$, для почти всех значений управляющего параметра первого элемента (т.е. для $0<\lambda_{1}<1$ ), системе необходимо практически одинаковое количество временных шагов для достижения своего равновесия. 
Рис. 3. Зависимость количества временных шагов $t$ от выбора регулирующих параметров $\lambda_{1}$ и $\lambda_{2}\left(c_{r}=25 / 4\right): \lambda_{2}=0,5,0<\lambda_{1}<1$. Источник: собственные вычисления

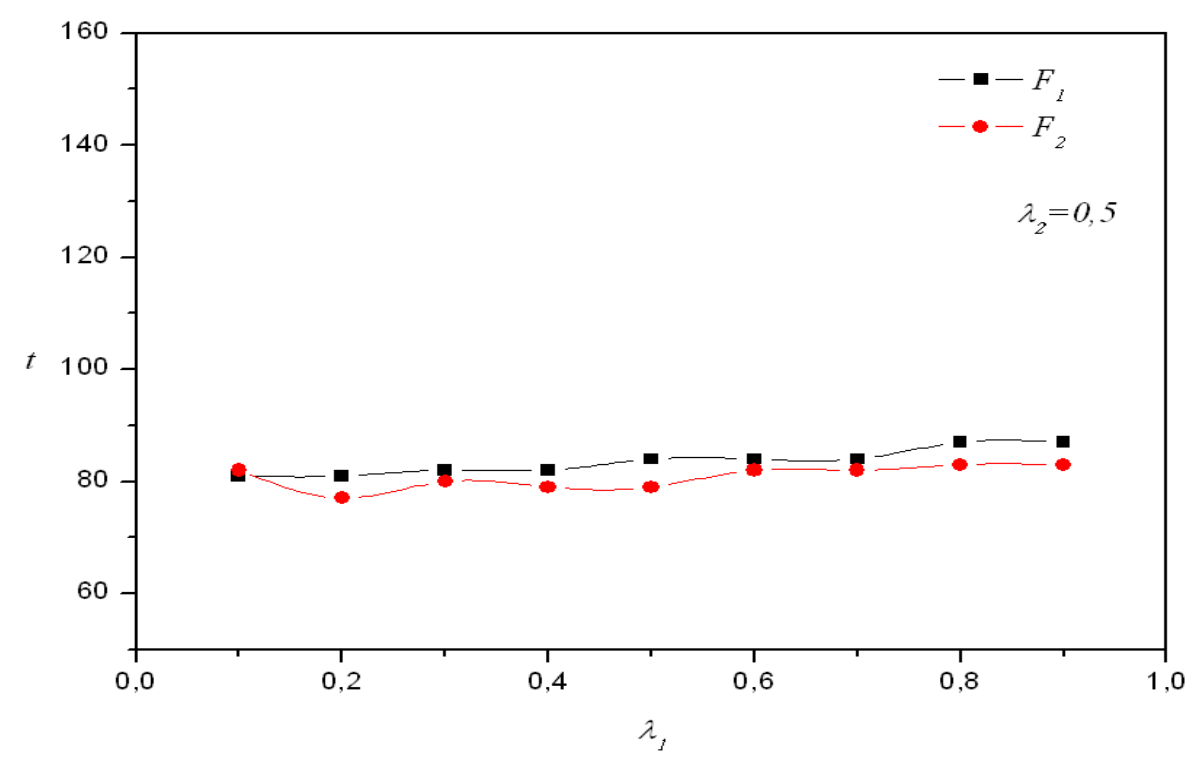

На рис. 4 показано, как элементы системы достигают равновесных значений своих исходных характеристик, с адаптивным регулированием неустойчивого процесса, при $\lambda_{1}=0,2, \lambda_{2}=0,5$. Как уже упоминалось выше, АС-метод начинает действовать с момента времени $t=50$.

Рис. 4. Изменение во времени исходных характеристик $q_{1}(t)$ и $q_{2}(t)$ элементов системы $F_{1}$ и $F_{2}$, соответственно, при значениях регулирующих параметров $\lambda_{1}=0,2, \lambda_{2}=0,5$. Источник: собственные вычисления

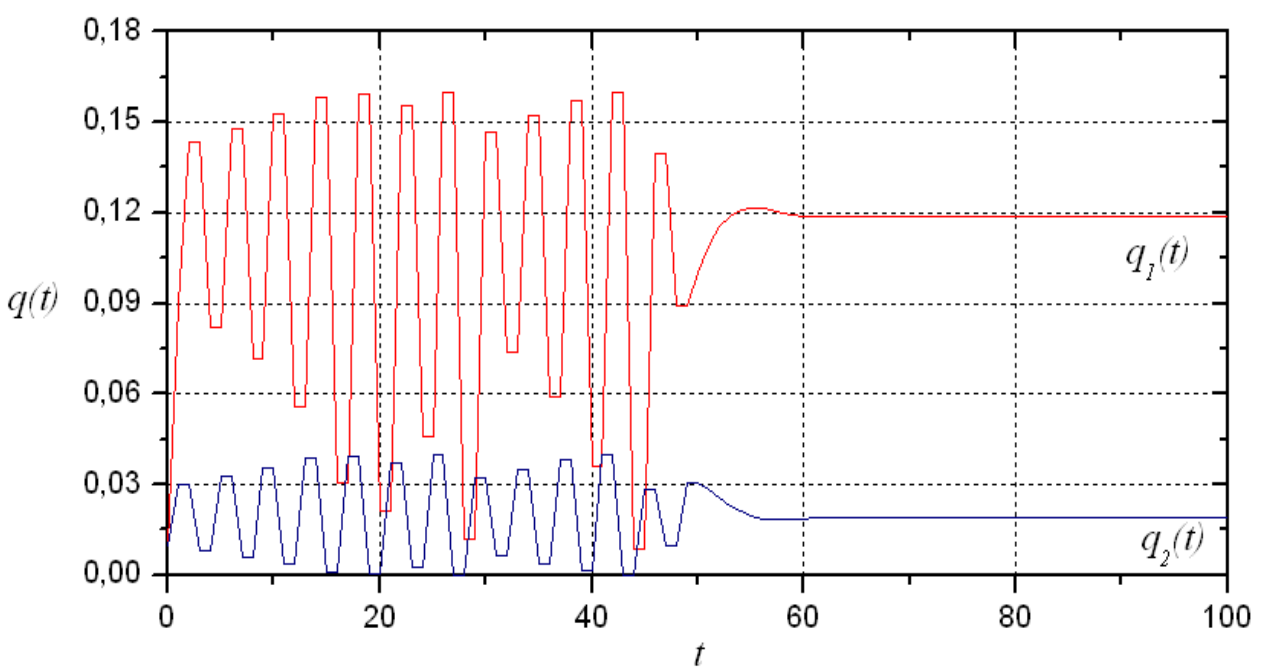


Такие же исследования были проведены и для прямых $\lambda_{2}=\left(1 / c_{r}\right) \lambda_{1}$ и $\lambda_{2}=c_{r} \lambda_{1}$, при $c_{r}=25 / 4$ (рис. 1), которые разделяют область устойчивости точки равновесия на области с действительными и комплексными собственными значениями. Результаты исследования показаны на рис. 5 и рис. 6.

Рис. 5. Зависимость количества временных шагов $t$ от выбора регулирующих

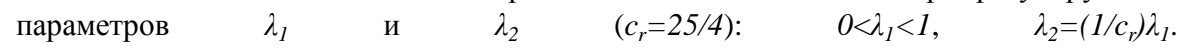

Источник: собственные вычисления

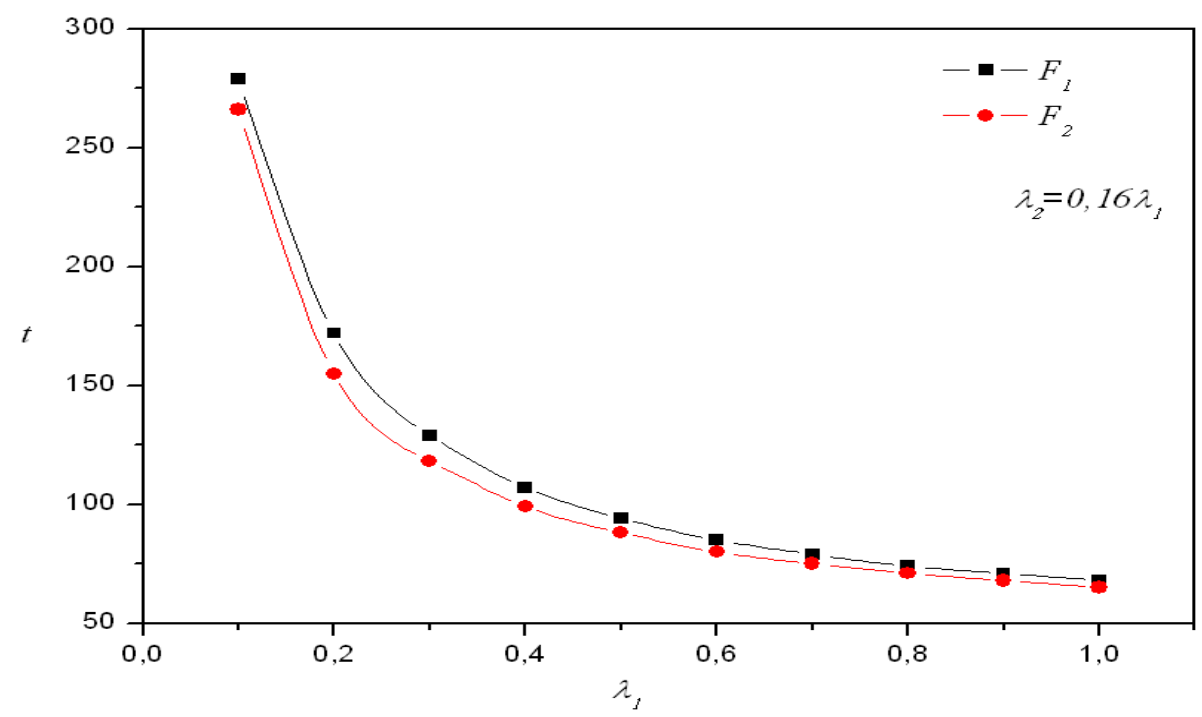

В этих исследованиях важно проанализировать также ситуацию, когда регулирующие параметры равны единице, что, как оказалось, дает интересные результаты. Из рис. 5 видно, что при выборе значений первого регулирующего параметра из допустимого интервала $0<\lambda_{1}<1$ (при этом значение второго параметра определялось из уравнения $\left.\lambda_{2}=\left(1 / c_{r}\right) \lambda_{1}\right)$, системе было необходимо меньшее количество временных шагов, чтобы прийти к равновесному состоянию, при $\lambda_{l}=1$, $\lambda_{2}=0,16\left(t_{1}=68-\right.$ для первого элемента, $t_{2}=65$ - для второго).

На рис. 6 показано подобную ситуацию при выборе второго управляющего параметра из интервала $0<\lambda_{2} \leq 1$ (при этом значения первого параметра определялись из уравнения $\left.\lambda_{1}=\left(1 / c_{r}\right) \lambda_{2}\right)$. В этом случае системе необходимо было меньше времени, чтобы прийти к равновесному состоянию, при $\lambda_{1}=0,16, \lambda_{2}=1\left(t_{1}=66, t_{2}=66\right)$. Очевидно, что количество временных шагов для обоих элементов здесь является наименьшим среди всех проведенных исследований для АС-метода (см. рис. 2-3, 5-6).

Кроме того, были проведены исследования для значений регулирующих параметров, когда $\lambda_{1}=1$ (при выборе второго параметра из интервала $0<\lambda_{2}<1$ ) или $\lambda_{2}=1$ (при выборе первого параметра из интервала $0<\lambda_{1}<1$ ). Полученные результаты показали, что на границе допустимой области при $\lambda_{l}=1$ (при выборе второго регулирующего параметра из интервала $\left.0<\lambda_{2}<1\right)$ элементы быстрее приходят в состояние равновесия при $\lambda_{1}=1, \lambda_{2}=0,2\left(t_{1}=66, t_{2}=64\right)$, а на границе $\lambda_{2}=1$ (при выборе значений первого регулирующего параметра из интервала $0<\lambda_{1}<1$ ) они достигают равновесия при $\lambda_{1}=0,2, \lambda_{2}=1\left(t_{1}=64, t_{2}=64\right)$. 
Рис. 6. Зависимость количества временных шагов $t$ от выбора регулирующих параметров $\lambda_{1} \quad$ и $\quad \lambda_{2} \quad\left(c_{r}=25 / 4\right): \quad 0<\lambda_{2}<1, \quad \lambda_{1}=\left(1 / c_{r}\right) \lambda_{2}$. Источник: собственные вычисления

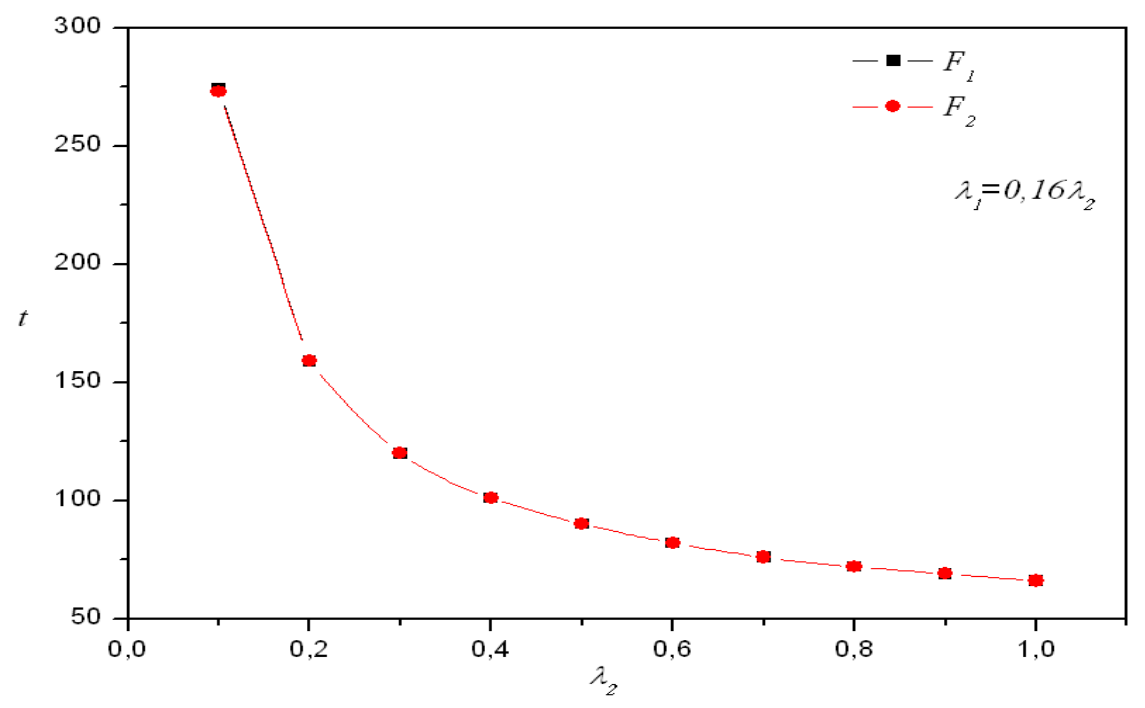

Рис. 7. Изменение во времени исходных характеристик $q_{1}(t)$ и $q_{2}(t)$ элементов системы $F_{1}$ и $F_{2}$, соответственно, при значениях регулирующих параметров $\lambda_{1}=0,2, \lambda_{2}=1$. Источник: собственные вычисления

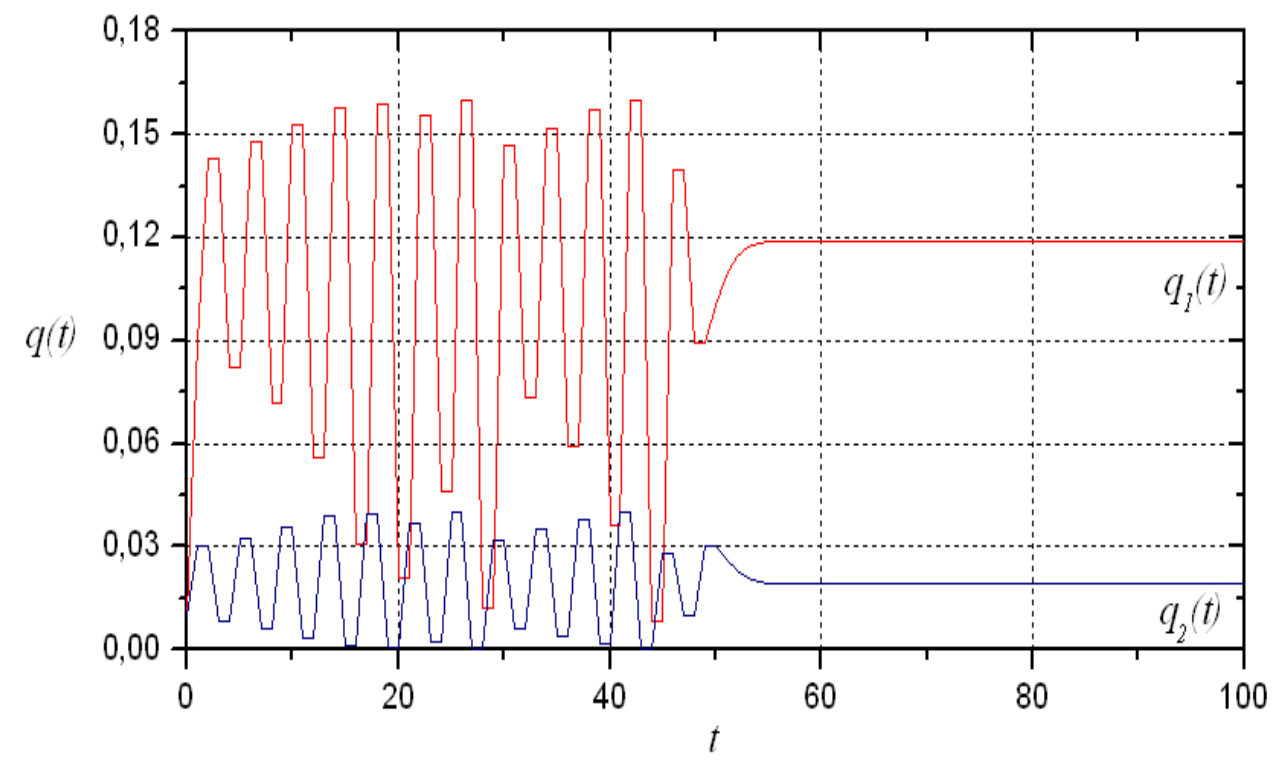

Как показано на рис. 7, элементы системы достигают равновесных значений своих исходных характеристик с адаптивным регулированием неустойчивого процесса при $\lambda_{1}=0,2, \lambda_{2}=1$. Ситуация, когда $\lambda_{1}=1, \lambda_{2} \neq 1$ (либо $\lambda_{2}=1, \lambda_{1} \neq 1$ ), означает, что первый элемент (либо второй элемент) не имеет возможности контролировать 
нестабильный процесс, а следовательно, процесс продолжается, как и в предыдущем периоде, без адаптивного регулирования. Предыдущие исследования показали, что один из конкурирующих элементов может самостоятельно стабилизировать неустойчивые колебания в системе, причем делает это значительно быстрее, чем в случае, когда процесс пытаются регулировать оба элемента этой системы одновременно.

Таким образом, проведенные исследования для АС-метода позволяют сделать вывод, подобный выводу в случае DFC-метода, а именно, в двухэлементных системах с конкуренцией, с использованием моделей Курно-Пу, для стабилизации хаотической динамики в системе целесообразно применять только одну управляющую функцию. Такое управление быстрее приводит систему к состоянию равновесия, по сравнению с применением двух управляющих функций, т.е. попытки двух элементов одновременно стабилизировать систему.

Именно поэтому применение метода адаптивного контроля, как и применение DFC-метода к структуре системы, также считается методом "индивидуального" контроля в неустойчивой системе. Один из элементов системы может самостоятельно, проанализировав ситуацию в системе, изменить ее состояние собственными действиями, фиксируя значение своей исходной характеристики в настоящем и прошлом периодах. Для дуополии это означает, что фирма-производитель может самостоятельно стабилизировать хаотичный рынок, наблюдая за собственными объемами производства в предыдущих периодах своей деятельности.

Сравнивая оба метода, следует также отметить, что были получены такие значения коэффициента обратной связи для DFСметода и значения регулирующих параметров для АС-метода, которые позволяют привести систему к состоянию равновесия практически за одинаковое количество временных шагов. Однако преимуществом DFC-метода является возможность управления также и параметром, характеризующим предельное поведение элементов системы, т.е. управляющие элементы внешней среды могут стабилизировать хаотическую динамику в системе путем воздействия на параметры предельного поведения ее конкурирующих элементов.

В случае дуополии управление параметрами системы можно рассматривать как метод регулирования со стороны правительства с целью стабилизации хаотичности на рынке. Правительство может ввести соответстующий налог с целью повышения предельных издержек одной из фирм (что может привести к снижению объемов ее производства), или выплатить дотацию с целью снижения предельных издержек более слабой из фирм (что приведет к увеличению объемов ее производства).

\section{5. ВЫВОДЫ}

Управление техническими и производственными системами с конкуренцией является одной из важнейших проблем, возникающих при их исследовании. Установление того факта, что устойчивость системы с конкуренцией существенно зависит от значения параметров предельного поведения ее элементов и требует применения эффективных методов контроля неустойчивой динамики, возникающей в таких системах. В работе был применен метод адаптивного контроля к управлению хаотичным поведением в системе с конкуренцией, состоящей из двух элементов (дуополии), с использованием модели Курно-Пу. 
На основе полученных теоретических результатов проведено ряд численных исследований, которые дают возможность сформулировать рекомендации по управлению нестабильными колебаниями в системе. В частности, определено значение регулирующих параметров, при которых хаотическая система стабилизируется за наименьшее количество временных шагов. Установлено, что адаптивный контроль является эффективным, если только один из элементов системы регулирует хаотическую динамику. Это означает, что один из регулирующих параметров системы равен единице: $\lambda_{1}=1, \lambda_{2} \neq 1$ (либо $\lambda_{2}=1, \lambda_{1} \neq 1$ ). В таком случае система быстро приходит в равновесное состояние.

Проведено сравнение метода адаптивного контроля и метода управления с обратной связью с задержкой, применяя их с целью урегулирования нестабильных колебаний показателей деятельности фирм на олигополистическом рынке, в частности, объемов выпуска их продукции. Установлено, что в случае применения как первого, так и второго методов для стабилизации хаотического рынка достаточно, чтобы только одна из фирм контролировала свои объемы производства. Количество необходимых для этого временных периодов для обоих методов отличается не существенно.

Исследованные модели управления можно использовать не только для стабилизации неустойчивой динамики на олигополистических рынках, но и во всех системах, элементы которых взаимосвязаны и конкурируют между собой относительно общего ограниченного ресурса. Это могут быть разного типа технические системы, информационные сети, информационно-телекоммуникационные, электроэнергетические и другие производственные структуры либо рынки. Достаточно эффективным может быть применение рассмотренных моделей управления в области информационных технологий, когда возникает конкурентная борьба за информационные потоки или ресурсы. Более детальное исследование возможных применений моделей управления типа Курно-Пу будет предметом дальнейших исследований.

\section{ЛИТЕРАТУРА}

[1] Chen L. , Chen G., Controlling chaos in an economic model, "Physica A" 374 (2007), s. 349-358.

[2] Puu T., Chaos in duopoly pricing, "Chaos, Solitons \& Fractals" 1 (1991), s. 573-581.

[3] Иващук Н., Кавалец И., Математические методы контроля нестабильных колебаний в конкурируюших системах, „Інформаційна безпека" 1 (2013), s. 12-24.

[4] Іващук Н.Л., Гнатів Б.В., Кавалець I.І., Побудова узагальненої моделі олігополії Курно-Пу та дослідження стійкості ї̈ точки рівноваги, „Журнал обчислювальної та прикладної математики” 2/112 (2013), s. 94-104.

[5] Костробій П.П., Алексєєв І.В., Хома І.Б., Гнатів Б.В., Кавалець I.І., Алексєєв В.І, Математичні моделі регулювання фінансових потоків, ред. П.П. Костробій, Растр-7, Львів 2012.

[6] Cournot A., Recherches sur les principes mathématiques de la théorie des richesses, Paris: Hachette 1838. 
[7] Iwaszczuk N., Kavalets I., Application of mathematical models in the study of oligopolistic markets, [w:] Zastosowania modeli matematycznych w ekonomii, finansach i bankowości, red. P. Pusz. Wydawnictwo Uniwersytetu Rzeszowskiego, Rzeszów 2012, s. 27-47.

[8] Iwaszczuk N., Kavalets I., Delayed feedback control method for generalized Cournot-Puu oligopoly model, [w:] Selected Economic and Technological Aspects of Management, red. N. Iwaszczuk. Wydawnictwo AGH w Krakowie, Kraków 2013, s. 108-123.

[9] Iwaszczuk N., Kavalets I., Generalized Cournot-Puu oligopoly model and stability of its equilibrium point, [w:] Materiały z konferencji ,Zarządzanie Przedsiębiorstwem - Teoria i Praktyka" (Kraków, 22-23 XI 2012), Wydawnictwo AGH w Krakowie, Kraków 2012, s. 1-16.

[10] Iwaszczuk N., Kavalets I., Oligopolistic market: stability conditions of the equilibrium point of the generalized Cournot-Puu model, "Econtechmod" 2/1 (2013), s. 15-22.

[11] Iwaszczuk N., Kavalets I., Some features of application the delayed feedback control method to Cournot-Puu duopoly model, "Econtechmod" 2/4 (2013), s. 29-38.

[12] Jakimowicz A., Stability of the Cournot-Nash equilibrium in standard oligopoly, "Acta Physica Polonica A" 121/2-B (2012), s. B-50-B-53.

[13] Matsumoto A., Controlling the Cournot-Nash Chaos, "Journal of Optimization Theory and Applications" 128/2 (2006), s. 379-392.

\section{ADAPTIVE CONTROL METHOD OF UNSTABLE DYNAMICS IN A TWO-}

ELEMENT SYSTEM WITH THE COMPETITION IN THE DUOPOLY CASE

Research of competition systems' dynamics is one of the current issues within the framework of systems and processes modeling; in particular, one of such issues is the fact that under certain conditions the systems show undesirable unsteady dynamics which can result in unforeseeable behaviour of the system's elements. Mathematical models of Cournot-Puu type give an opportunity to analyze such processes, as they take into account real conditions of the competition systems functioning as mentioned above.

Application of the DFC-method with the purpose of stabilizing unsteady behaviour of the systems with competition is expedient along with correlating it to equivalent methods, in particular, to the method of adaptive control - AC-method, basic principle of the latter being the hypothesis that expectations of the system's elements deviate from "naive" to "adaptive". The optimal value of original characteristic of the system element in a subsequent period which depends on the value of this characteristic in the current period, as well as on the weighted average expected value of the same original characteristic of a competitive element in the current period.

Conducted investigations allow us to draw a conclusion that for the 2-elements systems with competition it is reasonable to apply only one managing function for stabilizing the system chaotic dynamics. Such management restores the system to equilibrium faster as compared to application of two managing functions, i.e. attempts of two elements to stabilize the system simultaneously. For this particular reason application of the adaptive control method, as well as application of DFC-method, to the system structure is also considered to be the method of "individual" control in an unsteady system. 
The investigated management systems may be applied not only for stabilizing unsteady dynamics in oligopolistic markets, but also to all systems the elements of which are interrelated and compete for a limited resource.

Keywords: system with the competition, duopoly, Cournot-Puu model, equilibrium, unstable dynamics, adaptive control method.

\section{METODA ADAPTACYJNEJ KONTROLI NIESTABILNOŚCI DYNAMIKI W DWUELEMENTOWYM SYSTEMIE Z KONKURENCYJNOŚCIĄ NA PRZYKLADZIE DUOPOLU}

Jednym $\mathrm{z}$ aktualnych problemów $\mathrm{w}$ modelowaniu systemów i procesów jest badanie dynamiki funkcjonowania systemów z konkurencją, w szczególności ten fakt, że w pewnych warunkach pojawia się $\mathrm{w}$ nich niestabilna dynamika, która w dłuższej perspektywie czasu może doprowadzić do nieprzewidywanych zachowań elementów tychże systemów. Matematyczne modele typu Cournota-Puu tworzą możliwości przeprowadzania analizy takich procesów, ponieważ uwzględniają rzeczywiste warunki funkcjonowania wyżej wymienionych systemów z konkurencją.

Przy stosowaniu metody DFC, w celu ustabilizowania niestabilnego zachowania systemu z konkurencją, warto porównać ją z podobnymi metodami, między innymi $\mathrm{z}$ metodą sterowania adaptacyjnego - metodą AC, zasada działania której polega na założeniu, że oczekiwania elementów systemu zmieniają się od „naiwnych” do „adaptacyjnych". Optymalna wartość pierwotnej cechy charakterystycznej elementu systemu w następnym okresie zależy od wartości tej cechy w bieżącym okresie, a także od średniej ważonej wartości oczekiwanej tejże pierwotnej cechy elementu konkurencyjnego, również w bieżącym okresie.

$\mathrm{Na}$ podstawie przeprowadzonych badań można wyciągnąc wniosek, że aby ustabilizować chaotyczną dynamikę w dwuelementowych systemach z konkurencją, należy zastosować tylko jedną funkcję sterowania. Taki sposób sterowania szybciej doprowadzi system do równowagi, w porównaniu ze stosowaniem dwóch funkcji sterowania, tzn. próby stabilizowania systemu ze obydwu jego elementów jednocześnie. Dlatego zastosowanie metody sterowania adaptacyjnego, zarówno jak i zastosowanie metody DFC do struktury systemu, uważa się też za metodę , indywidualnej” kontroli w niestabilnym systemie.

Zbadane modele można wykorzystywać nie tylko do stabilizacji niestabilnej dynamiki na rynkach oligopolistycznych, lecz także we wszystkich tych systemach, elementy których są ze sobą powiązane i konkurują ze sobą względem wspólnego ograniczonego zasobu. Słowa kluczowe: system z konkurencją, duopol, model Cournota-Puu, punkt równowagi, niestabilna dynamika, metoda adaptacyjnego sterowania.

\section{DOI:10.7862/rz.2016.hss.42}

Przesłano do redakcji: kwiecień 2016

Przyjęto do druku: wrzesień 2016 